\title{
Origins of Portal Hypertension in Nonalcoholic Fatty Liver Disease
}

\section{Citation}

Baffy, Gyorgy. 2018. "Origins of Portal Hypertension in Nonalcoholic Fatty Liver Disease." Digestive Diseases and Sciences (January 22). doi:10.1007/s10620-017-4903-5.

\section{Published Version}

10.1007/s10620-017-4903-5

\section{Permanent link}

http://nrs.harvard.edu/urn-3:HUL.InstRepos:34814070

\section{Terms of Use}

This article was downloaded from Harvard University's DASH repository, and is made available under the terms and conditions applicable to Other Posted Material, as set forth at http:// nrs.harvard.edu/urn-3:HUL.InstRepos:dash.current.terms-of-use\#LAA

\section{Share Your Story}

The Harvard community has made this article openly available.

Please share how this access benefits you. Submit a story.

\section{Accessibility}




\title{
Origins of Portal Hypertension in Nonalcoholic Fatty Liver Disease
}

\author{
Gyorgy Baffy ${ }^{1}[\mathbb{D}$
}

Received: 21 November 2017 / Accepted: 26 December 2017

(C) This is a U.S. Government work and not under copyright protection in the US; foreign copyright protection may apply 2018

\begin{abstract}
Nonalcoholic fatty liver disease (NAFLD) advanced to cirrhosis is often complicated by clinically significant portal hypertension, which is primarily caused by increased intrahepatic vascular resistance. Liver fibrosis has been identified as a critical determinant of this process. However, there is evidence that portal venous pressure may begin to rise in the earliest stages of NAFLD when fibrosis is far less advanced or absent. The biological and clinical significance of these early changes in sinusoidal homeostasis remains unclear. Experimental and human observations indicate that sinusoidal space restriction due to hepatocellular lipid accumulation and ballooning may impair sinusoidal flow and generate shear stress, increasingly disrupting sinusoidal microcirculation. Sinusoidal endothelial cells, hepatic stellate cells, and Kupffer cells are key partners of hepatocytes affected by NAFLD in promoting endothelial dysfunction through enhanced contractility, capillarization, adhesion and entrapment of blood cells, extracellular matrix deposition, and neovascularization. These biomechanical and rheological changes are aggravated by a dysfunctional gut-liver axis and splanchnic vasoregulation, culminating in fibrosis and clinically significant portal hypertension. We may speculate that increased portal venous pressure is an essential element of the pathogenesis across the entire spectrum of NAFLD. Improved methods of noninvasive portal venous pressure monitoring will hopefully give new insights into the pathobiology of NAFLD and help efforts to identify patients at increased risk for adverse outcomes. In addition, novel drug candidates targeting reversible components of aberrant sinusoidal circulation may prevent progression in NAFLD.
\end{abstract}

Keywords Sinusoidal homeostasis $\cdot$ Endothelial dysfunction $\cdot$ Hepatic venous pressure gradient $\cdot$ Intrahepatic vascular resistance

\section{Introduction}

Nonalcoholic fatty liver disease (NAFLD) is estimated to affect more than a billion individuals worldwide, including $25-30 \%$ of the US population $[1,2]$. NAFLD has a strong association with visceral obesity, insulin resistance, and endothelial dysfunction [3]. The spectrum of NAFLD ranges from steatosis to steatohepatitis with a variable degree of liver fibrosis. Liver-related mortality is not substantially affected by steatosis, while $10-25 \%$ of steatohepatitis will progress into cirrhosis over 8-14 years with an increased risk of developing portal hypertension (PHT), liver failure,

Gyorgy Baffy

gbaffy@bwh.harvard.edu

1 Department of Medicine, VA Boston Healthcare System and Brigham and Women's Hospital, Harvard Medical School, 150 South Huntington Avenue, Room 6A-46, Boston, MA 02130, USA and hepatocellular carcinoma [4]. While substantial evidence indicates that fibrosis is the ultimate predictor of longterm outcomes in patients with NAFLD [4-6], risk factors associated with disease progression are not fully identified.

PHT is responsible for most complications associated with advanced NAFLD. When related to chronic liver disease, PHT as a clinical manifestation of increased portal venous pressure (PVP) is generally regarded as a late consequence of parenchymal and vascular remodeling of the cirrhotic liver with extensive fibrosis as a key process of sinusoidal obstruction $[7,8]$. However, observations made in clinical and experimental NAFLD suggest that PVP may begin to rise when fibrosis is far less advanced or absent $[9,10]$. The significance of these early changes in sinusoidal pathobiology and the role of increased PVP in fibrosis progression and clinical outcomes of NAFLD remains unclear [11, 12]. It seems therefore timely to review current knowledge about the development of PHT across the entire spectrum of NAFLD. Identifying the cellular and molecular 
origins of increased PVP in NAFLD may improve our understanding of the pathophysiology, assist risk stratification for disease progression, and define new therapeutic targets.

\section{General Aspects of Portal Hypertension}

The liver receives one-fifth of cardiac output from a dual blood supply, two-thirds of which come from the portal vein and one-third from the hepatic artery. The portal vein collects partially deoxygenated, nutrient-rich blood from the splanchnic area, while the hepatic artery delivers highly oxygenated blood to the liver. Terminal branches of the portal vein drain into the sinusoids joined by terminal hepatic arterioles to form a unique capillary network [13]. Experimental studies indicate that the hydrostatic pressure in hepatic arterioles is 20- to 40-fold higher than in terminal portal venules $[14,15]$. Thus, arteriolar inflow needs to be efficiently regulated to prevent shear stress in the liver sinusoids, which are low-pressure, low-flow vascular channels linking the periportal area of portal inflow (zone 1) to the centrilobular area of central vein outflow (zone 3) (Fig. 1a).

According to Ohm's law, vascular pressure is a function of vascular resistance and vascular flow $(\Delta P=R \times F)$ $[7,8]$. Depending on the site of impediment, PHT can be classified as pre-sinusoidal, sinusoidal, or post-sinusoidal. Sinusoidal PHT is the most common form, which may complicate cirrhosis of any etiology [7]. In cirrhosis, sinusoidal
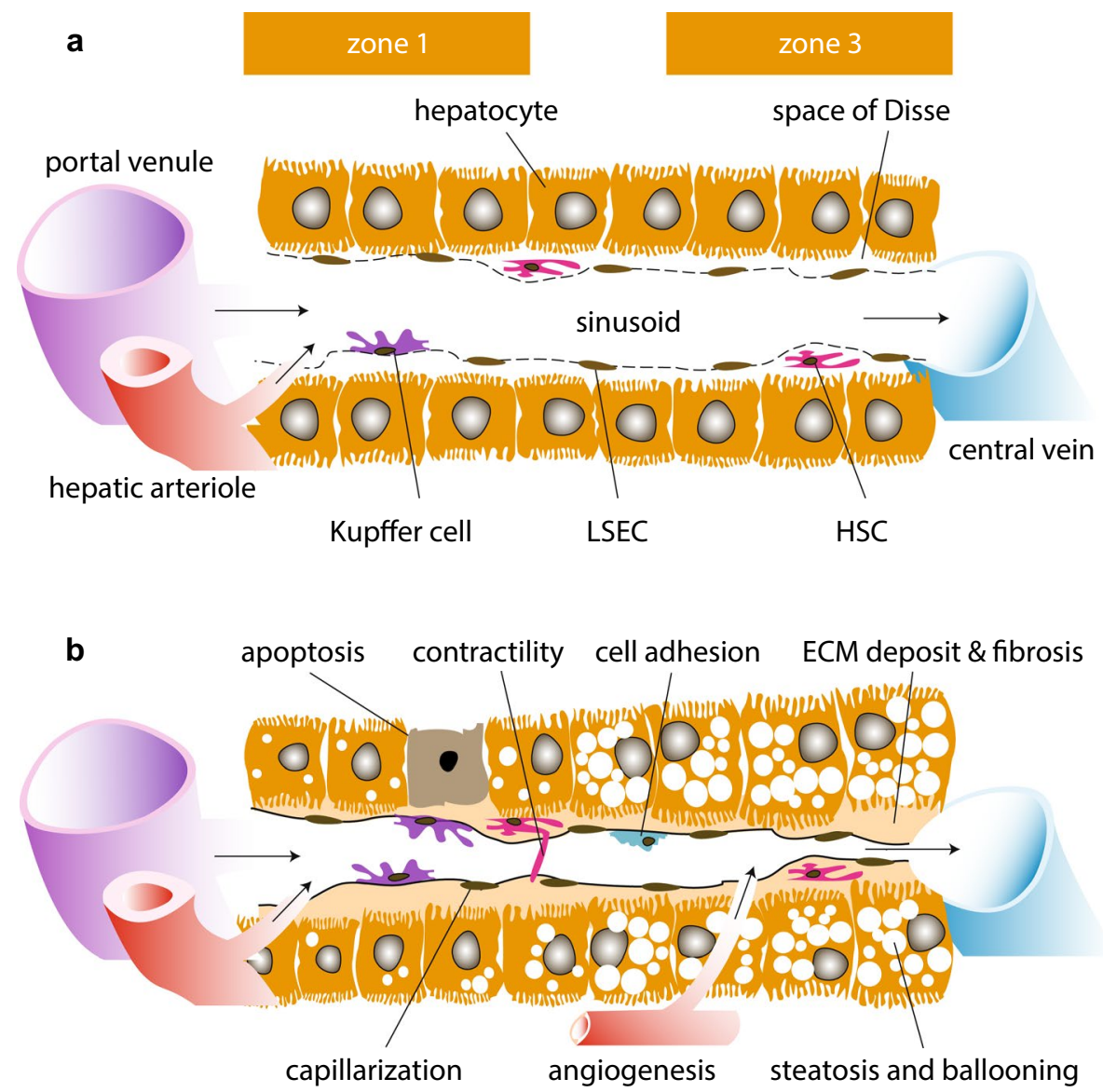

Fig. 1 Components of impaired sinusoidal homeostasis in NAFLD. a Schematized microcirculatory unit of normal liver with a portal venule and a hepatic arteriole merging into a sinusoid that drains to a central vein. Sinusoidal flow from the periportal (zone 1) to the centrilobular area (zone 3) is indicated by arrows. Hepatocytes are facing the space of Disse separated from the sinusoidal bloodstream by the fenestrated plasma membrane of liver sinusoidal endothelial cells (LSECs). Hepatic stellate cells (HSCs) are located within the space of Disse, while Kupffer cells reside within the sinusoids. b In NAFLD, enlarged fatty hepatocytes (steatosis) impede sinusoidal flow, first in zone 3 and then increasingly in the entire lobule, resulting in shear stress. LSECs respond by facilitating adhesion of blood cells and losing inhibitory control over activation of HSCs and vasoconstriction, further constraining the sinusoidal space. Due to lipotoxicity, hepatocellular ballooning develops resulting in additional sinusoidal compression. In response to these structural and functional changes, LSECs become defenestrated, develop a basement membrane (capillarization), and deposit extracellular matrix (ECM) within the space of Disse, limiting macromolecular/solute exchange and causing relative hypoxia. Hepatocellular injury and death (apoptosis) represent danger signals to Kupffer cells advancing inflammation and additional cell-cell interactions, which induce fibrosis and angiogenesis, further compromising sinusoidal flow and contributing to disease progression in NAFLD 
architecture becomes grossly distorted leading to increased intrahepatic vascular resistance (IHVR) accompanied by vasoregulatory changes in hepatic and systemic circulation. By contrast, the term "noncirrhotic PHT" refers to diverse conditions in which elevated portal venous pressure develops in the absence of cirrhosis [16]. Besides the obvious prehepatic and post-hepatic vascular conditions, noncirrhotic portal hypertension may result from disorders of sinusoidal obstruction such as granulomatous liver disease and nodular regenerative hyperplasia [17]. According to current terminology, increased PVP in NAFLD that may develop prior to cirrhosis is not classified as noncirrhotic PHT.

PVP is traditionally measured by retrograde occlusion of a hepatic vein tributary with a balloon-tipped central vein catheter, which detects wedged hepatic venous pressure (WHVP) [18]. Using umbilical vein pressure as reference, WHVP in cirrhotic patients was found to be almost identical to PVP, and the pressure difference between wedged and free-floating catheter positions defining the hepatic venous pressure gradient (HVPG) became a widely accepted measure of PHT [19, 20]. Thus, an HVPG of $5 \mathrm{~mm} \mathrm{Hg}$ or more indicates PHT and an HVPG of $10 \mathrm{~mm} \mathrm{Hg}$ or more is defined as clinically significant PHT, predicting the development of esophageal varices and other complications [21, 22]. However, the biological and clinical impact of mild PHT (i.e., HVPG ranging from 5 to $10 \mathrm{~mm} \mathrm{Hg}$ ) on the course of NAFLD is incompletely understood.

\section{Portal Hypertension in Noncirrhotic NAFLD}

It has long been known from animal studies that steatosis may be associated with increased PVP. In a classic experiment performed more than 40 years ago, the impact of choline-deficient diet on PVP was analyzed in rats that developed fatty liver, fatty liver with fibrosis or fatty cirrhosis [23]. Surprisingly, increased PVP, decreased portal blood flow, and sinusoidal narrowing without visible abnormalities in pre- and post-sinusoidal vessels were already present in the fatty liver group, suggesting that steatosis alone is sufficient to generate PHT in this model [23]. In another study using Zucker obese rats characterized by massive steatosis but no cirrhosis, total hepatic blood flow and portal venous flow were reduced by 35 and 38\%, respectively, consistent with increased IHVR compared to nonsteatotic animals [24].

Similarly, hemodynamic alterations consistent with increased IHVR have been detected in human fatty liver. When assessed by Doppler ultrasonography, PHT is characterized by deceleration of portal vein flow and a compensatory increased flow in the hepatic artery [25]. Accordingly, portal vein pulsatility index (defined as [peak maximum velocity - peak minimum velocity]/peak maximum velocity) is low due to diminished peak maximum velocity in the portal vein, while hepatic arterial resistance index (defined as [peak systolic velocity - end diastolic velocity]/peak systolic velocity) is low due to increased end diastolic velocity in the hepatic artery. The arterioportal flow ratio combines these opposing circulatory changes and provides another correlate of portal venous pressure. In a study from Turkey comparing 35 noncirrhotic NAFLD patients with 35 healthy controls, portal vein pulsatility index and mean flow velocity inversely correlated with the ultrasonographic grade of steatosis [26]. In another study from Japan, hepatic circulation was analyzed in a cohort of 121 patients with biopsy-proven NAFLD featuring variable degree of fibrosis but no cirrhosis [27]. These authors found that the arterioportal flow ratio correlated with the histological degree of liver fibrosis and higher readings were also detected in some patients with F0 and F1 fibrosis [27].

In a recent observational study, the prevalence of PHT was analyzed in a cohort of 354 patients undergoing liver biopsy for NAFLD staging [10]. A total of 100 patients were found to have esophageal varices, encephalopathy, splenomegaly or ascites, all consistent with clinically significant PHT. While cirrhosis was confirmed in most cases, fibrosis was mild or absent in 12 patients (12\%). Moreover, extent of steatosis was the only difference when comparing noncirrhotic patients with and without PHT. These findings suggest that even clinically significant PHT may develop in some cases of NAFLD without cirrhosis if steatosis is sufficiently severe [10]. However, generalizability of this study is limited as the diagnosis of PHT was established by surrogate clinical, laboratory, and imaging markers.

More definitive evidence for the presence of PHT in noncirrhotic NAFLD has been gained from studies utilizing PVP measurements. In a recent work, $\mathrm{HVPG}$ exceeded $5 \mathrm{~mm} \mathrm{Hg}$ in 8 out of 40 obese patients $(20 \%)$ with no cirrhosis verified by transjugular liver biopsy, while one patient had an HVPG $>10 \mathrm{~mm} \mathrm{Hg}$ indicating clinically significant PHT [28]. Another analysis of 50 patients with noncirrhotic NAFLD found that mean fibrosis scores of 27 subjects with normal PVP and 23 subjects with elevated PVP were similar, while steatosis, waist circumference, and HOMA-IR were all independently predictive of higher PVP [9]. These observations (within the limits of sampling error in liver biopsy) provide additional evidence that PHT may occur in NAFLD when steatosis is either the sole histological feature or minimal/ mild fibrosis is present, with excess visceral adipose tissue and insulin resistance playing a significant role in this process [9]. 


\section{Cellular and Molecular Mechanisms of Portal Hypertension in NAFLD}

\section{Steatosis and Hepatocellular Ballooning}

Several mechanisms may impair sinusoidal hemostasis and contribute to the gradual rise of IHVR in NAFLD before cirrhosis develops (Fig. 1b). Hepatocellular enlargement due to lipid accumulation in NAFLD is probably the earliest mechanical barrier to sinusoidal flow as lipid-laden cells reduce sinusoidal space by as much as $50 \%$ compared with normal liver [29]. Histological analysis of 545 liver specimens obtained from patients enrolled by the Nonalcoholic Steatohepatitis Clinical Research Network indicated that mild steatosis is predominantly centrilobular before the pattern becomes pan-acinar in severe steatosis or steatohepatitis [30]. These observations are consistent with enhanced lipogenesis detected in zone 3 hepatocytes and suggest that sinusoidal flow in the earliest stages of NAFLD is primarily perturbed in the centrilobular region [31].

Sustained accumulation of lipid molecules and their derivatives in hepatocytes may cause lipotoxicity, affecting gene transcription, cellular signaling pathways, reactive oxygen species (ROS) formation, endoplasmic reticulum stress, and energetic vulnerability with cell death (lipoapoptosis) as a possible outcome [32]. Hepatocellular ballooning as a characteristic feature of steatohepatitis is related to lipotoxicity [30,33]. Lipid vacuoles in ballooned hepatocytes contain increased amounts of oxidized phosphatidylcholine along with altered expression of fat droplet-associated proteins such as perilipin-2 [34]. These changes are accompanied by dilation of the endoplasmic reticulum and disorganization of the cytoskeleton, which becomes deficient in cytokeratin 18 [35]. Ballooned hepatocytes may have a diameter 1.5-2 times of their normal counterparts, further contributing to sinusoidal compression and elevated IHVR [33]. Notably, ballooned hepatocytes increasingly produce sonic hedgehog, a key signaling molecule implicated in subsequent development of liver fibrosis [36].

\section{Liver Cell-Cell Interactions}

Nonparenchymal liver cells interact with fatty hepatocytes through complex endocrine, paracrine, and autocrine regulatory loops that further contribute to the disruption of sinusoidal homeostasis and may increase IHVR in NAFLD (Fig. 2). Liver sinusoidal endothelial cells (LSECs) form a sinusoidal lining with holes up to $200 \mathrm{~nm}$ and no regular basement membrane [37]. The fenestrated endothelium of liver sinusoids is organized into sieve plates that allow macromolecules and cellular projections to enter the space of Disse, which separates sinusoidal blood from hepatocytes. Through the activity of endothelial nitric oxide synthase (eNOS), LSECs are the main source of nitric oxide (NO), a key vasodilator molecule regulating sinusoidal flow in the liver [37]. Normally, endothelial NO production is regulated by Kruppel-like factor 2 (KLF2), a transcription factor that also curbs the expression of vasoconstrictor mediators and becomes increasingly upregulated in LSECs exposed to shear stress [38]. This vasoregulatory response is impaired in liver injury, resulting in increased contractility and IHVR [37, 39]. Under these conditions, LSECs increasingly produce vascular cell adhesion molecule 1 (VCAM-1) and other factors that promote adherence of portal blood cells to the sinusoidal endothelium [39]. Some sinusoids may be narrow enough to be entirely obliterated by trapped cells, which is more likely to occur in the centrilobular region, accentuating the impact of steatosis [40]. Steatosis induced in Wistar Kyoto rats by the administration of cafeteria diet rich in saturated fatty acids over 1 month was associated with blunted activation of eNOS by insulin-induced phosphorylation at the Ser1176 residue indicating insulin resistance and impaired capacity of LSECs to release NO under these conditions [41]. Portal perfusion pressure in these livers was higher, and the vasodilator response to acetylcholine was diminished. Since these changes were observed in the absence of inflammation or fibrosis, the findings suggest that endothelial dysfunction is an early feature associated with steatosis in NAFLD [41].

Hepatic stellate cells (HSCs) are located in the space of Disse with long cellular projections wrapped around one or more sinusoids, similar to the arrangement of pericytes in the systemic circulation [42]. In normal conditions, HSCs remain quiescent in part because of the tonic inhibition of NO released from LSECs [37]. In response to liver injury, HSCs are activated through paracrine or autocrine mechanisms that involve platelet-derived growth factor (PDGF), insulin-like growth factor (IGF), endothelin-1, eicosanoid derivatives, and extracellular matrix components such as fibronectin [42]. Upregulation of the smooth muscle proteins actin and myosin increases the contractility of activated HSCs, further restricting sinusoidal flow [43]. Experimental observations in cirrhotic rat livers suggest that this reversible component accounts for $20-30 \%$ of IHVR [44]. In the absence of cirrhosis, however, this process may not be sufficiently synchronized across larger regions of the liver to yield clinically significant PHT [7].

Kupffer cells are liver macrophages that recognize molecular danger signals derived from the portal circulation and implicated in the pathogenesis of NAFLD [45]. Exposure of Kupffer cells to pathogen-associated 


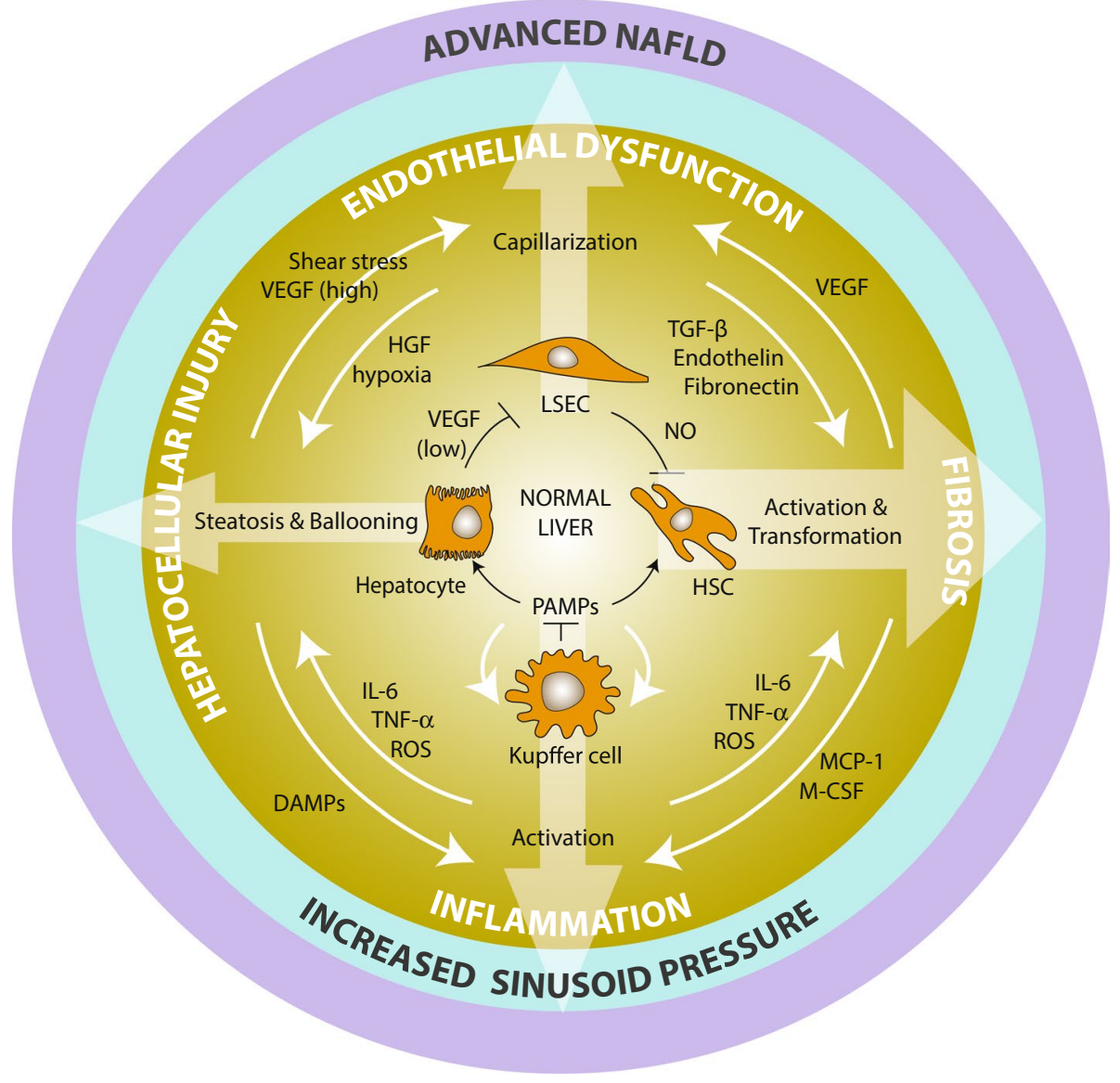

Fig. 2 Liver cell-cell interactions and the development of portal hypertension in NAFLD. In normal liver (center), sinusoidal homeostasis depends on low-level release of vascular endothelial growth factor (VEGF) by hepatocytes, helping liver sinusoidal endothelial cells (LSECs) to remain differentiated and to generate nitric oxide (NO), which inhibits activation of hepatic stellate cells (HSCs), while Kupffer cells remove bacterial endotoxin (LPS) and other portalderived pathogen-associated molecular patterns (PAMPs) to curb their effects on liver injury, inflammation and fibrosis (black arrows). In NAFLD, hepatocytes and nonparenchymal liver cells increasingly acquire different phenotypes and interact via multiple mediators (small white arrows). Increased VEGF production in addition to shear stress by steatotic and ballooning hepatocytes result in LSEC capillarization, hepatocyte growth factor (HGF) secretion and impaired

molecular patterns (PAMPs) stimulates pattern recognition receptors such as Toll-like receptor 4 (TLR4), which binds lipopolysaccharide (LPS) or endotoxin, a major cell wall component of Gram-negative bacteria found in the gastrointestinal tract [46]. Recently, the complex relationship of gut microbiota with sinusoidal homeostasis in NAFLD has been increasingly appreciated and will be further discussed below [47, 48]. Kupffer cells are also activated by endogenous damage-associated molecular patterns (DAMPs) of injured and dying hepatocytes and by HSCs via chemokines such as macrophage colony-stimulating factor (M-CSF) and monocyte chemoattractant protein-1 perfusion (hypoxia), further aggravating hepatocellular injury that activates Kupffer cells via damage-associated molecular patterns (DAMPs), adding to the impact of PAMPs with generation of reactive oxygen species (ROS) and pro-inflammatory cytokines such as interleukin-6 (IL-6) and tumor necrosis factor- $\alpha$ (TNF- $\alpha$ ). These mediators activate HSCs, which are also stimulated by capillarized LSECs via transforming growth factor- $\beta$ (TGF- $\beta$ ), endothelin, and fibronectin, leading to secretion of collagen, VEGF, and chemokines such as macrophage colony-stimulating factor (M-CSF) and monocyte chemoattractant protein-1 (MCP-1). Multiple feed-forward cycles in this complex cross talk promote increased sinusoid pressure and histological features of NAFLD with variable impact on disease progression (large white arrows)

(MCP-1) [42]. Activated Kupffer cells release chemokines, eicosanoid derivatives, and reactive oxygen species (ROS) that invite additional cell-cell interactions and escalate the liver inflammatory response [49]. In contrast to the classic (M1) activation pathway, alternative (M2) activation of Kupffer cells may restrain inflammation, indicating a complex role in the pathogenesis of liver disease [45]. In a cohort of obese patients undergoing transjugular liver biopsy, HVPG correlated with higher M1/M2 ratios of portal macrophages inferred from the ratios of pro- and anti-inflammatory cytokines in liver circulation, identifying inflammation as a predictor of PHT [28]. 


\section{Sinusoidal Capillarization and Fibrosis}

In response to liver injury, LSECs undergo progressive defenestration and form a basement membrane in a process termed capillarization [50]. Capillarized LSECs begin to deposit extracellular matrix proteins such as fibronectin and laminin in the space of Disse [37]. These morphological changes impair hepatic perfusion and hypoxia, leading to increased activation of hypoxia-inducible factors (HIFs) implicated in the transcriptional regulation of genes involved in angiogenesis, metabolic adaptation, cell survival and proliferation [51]. Several groups described sinusoidal capillarization as an early manifestation of endothelial dysfunction in experimental NAFLD [41, 52]. These changes were observed as early as within 1 week after the initiation of choline-deficient, L-amino acid-defined diet, and capillarization has been considered a "gatekeeper" in the progression to steatohepatitis, occurring before the concerted activation of Kupffer cells and HSCs [52].

When HSCs are activated, collagen begins to accumulate in the space of Disse, while hepatocellular tissue plates initially maintain their structure [42]. Collagen deposition in NAFLD originates in zone 3 as perivenular fibrosis with a subtle pericellular component before extending into other parts of the liver lobule [53]. With further progression, portal fibrosis develops and increasingly dense fibrous bridges or septae grow between neighboring portal tracts and across the sinusoids [54]. This extensive fibrosis, along with cycles of hepatocellular destruction and regeneration, results in the formation of cirrhotic nodules [42]. Septal thickness and small nodule size have been the only independent predictors of clinically significant PHT in a study of 43 patients with cirrhosis of different etiologies [55], suggesting that steatosis or steatohepatitis may not further contribute to PHT at this advanced stage of liver disease.

\section{Hepatic Neovascularization and Altered Splanchnic Hemodynamics}

There is increasing evidence that neovascularization is a key element in the progression of NAFLD [56, 57]. Formation of new blood vessels in chronic liver disease is linked to the advancement of fibrosis, indicating a close interplay between LSECs and HSCs. Vascular endothelial growth factor (VEGF) is the master regulator of this process, mediating both pro-fibrogenic and pro-angiogenic signals and supported by HIF activation in hypoxic areas [57]. Importantly, serum VEGF levels of patients with steatosis and steatohepatitis are higher compared to healthy controls [56]. Moreover, in animal models of genetic $(\mathrm{db} / \mathrm{db})$ and methionine-choline-deficient diet (MCDD)-induced NAFLD, increased VEGF levels occur as early as 3 days into steatosis before steatohepatitis of fibrosis develops [56].
Intralobular arterioles occasionally drain to sinusoids midway between zones 1 and 3 in the classic liver lobule [58]. The impact of these "arterial twigs" on sinusoidal flow is incompletely understood, although they may represent areas of higher pressure [59]. In a recent review of liver biopsies from patients with steatohepatitis, centrilobular arteries were common in cases with higher-stage fibrosis and occurred in $60 \%$ of livers with stage $1 \mathrm{~b}$ (moderate zone 3 perisinusoidal) fibrosis [60]. Further studies may determine whether these vessels cause increased sinusoidal shear stress, representing self-perpetuation in the pathogenesis of PHT in NAFLD.

Recent research indicates that steatotic hepatocytes may release microparticles that mediate angiogenesis [61]. Exposure of human endothelial cells to supernatant of hepatocytes treated with saturated fatty acids mimicked the cellular response to VEGF, depended on endothelial uptake of microparticles in the range of $100-1000 \mathrm{~nm}$, and required lipid rafts and vanin-1 (VNN1), a plasma membraneanchored ectoenzyme involved in cell adhesion and trafficking [61]. VNN1-containing microparticles isolated from the blood of mice with MCDD-induced steatohepatitis replicated the angiogenic effect. Moreover, these microparticles occurred in the perisinusoidal space and showed annexin $\mathrm{V}$ positivity, suggesting a critical role in linking lipotoxicity and angiogenesis [61].

Splanchnic vasodilation is commonly associated with PHT in cirrhosis. Many vasoactive substances such as NO, glucagon, bile salts, platelet-activating factor, calcitonin gene-related peptide, atrial natriuretic peptide, adrenomedullin, and endocannabinoids have been implicated in arteriolar vasodilation in the visceral vascular bed that drains into portal circulation and causes increased portal inflow [7, 57]. These mediators may reach higher splanchnic concentrations due to impaired liver metabolism or increased portosystemic shunting [62]. Recent findings suggest that splanchnic vasodilation in NAFLD may contribute to increased PVP well before the development of cirrhosis. Mesenteric arterial and portal venous flow was higher in rats with simple steatosis induced by MCDD, underscoring the role of systemic metabolic and hormonal factors in sinusoidal vasoregulation [63].

\section{Dysbiosis and the Gut-Liver Axis}

The bidirectional relationship of gut microbiota with chronic liver disease is documented as the gut-liver axis with an important role in the pathophysiology of NAFLD [48]. Disruption in the makeup of gut microbiota (dysbiosis) may occur in response to dietary modifications, gastrointestinal motility disorders, medications or other environmental factors [64]. Recent evidence indicates that these changes occur in NAFLD and have potentially adverse effects on liver physiology. Altered intestinal bile acid metabolism impairs 
hepatic and extrahepatic signaling pathways that include the farnesoid X receptor (FXR) and the G-protein-coupled bile salt receptor TGR5, which normally mediate anti-steatotic, anti-inflammatory, and anti-fibrotic effects $[65,66]$. Moreover, weakening of the intestinal epithelial barrier may promote translocation of PAMPs into the portal circulation, stimulating the liver inflammatory response and disturbing sinusoidal homeostasis [48]. In turn, NAFLD progression is associated with increased systemic release of pro-inflammatory cytokines, amplifying the impact of dysbiosis and further impairing the function of intestinal tight junctions [67]. In cirrhotic patients with clinically significant PHT, we found distinct changes in duodenal epithelial morphology that become only apparent by confocal laser endomicroscopy and include increased columnar cell height and microvessel aberrations [68]. The importance of these changes in the pathophysiology of gut-liver axis remains unclear. However, there is increasing evidence that the vicious cycle of gut-liver dysfunction can be interrupted by manipulating the gut microbiota. Intestinal decontamination by rifaximin in mice following bile duct ligation reduces liver fibrosis, angiogenesis, and PHT in association with diminished activation of the LPS/TLR4 pathway and fibronectin production, which is limiting the cross talk between HSCs and LSECs [69]. Thus, modulation of the gut-liver axis may prove to be a helpful strategy for preventing and managing NAFLDassociated PHT [70, 71].

\section{Assessment of the Portal Venous Pressure in NAFLD}

There have been significant efforts to find novel approaches for the assessment of increased PVP and replace traditional HVPG measurements that are invasive and performed only in specialized centers. Current strategies for the noninvasive appraisal of PHT include the use of various serum biomarkers, imaging studies, endoscopy, and liver stiffness measurements alone or in combination (Table 1). The diagnostic performance of these methods in identifying patients with clinically significant PHT has been discussed extensively elsewhere [72-76]. It is important to note that these noninvasive methods have been primarily developed to predict the extent of liver fibrosis and very little is known about their ability to detect PHT that may precede cirrhosis and remains below the level of clinical significance (HVPG in the range of $5-10 \mathrm{~mm} \mathrm{Hg}$ ). While risk stratification and preventive care in NAFLD could potentially benefit from the detection of mildly increased PVP, no such method has been validated in noncirrhotic patient populations.

Subharmonic aided pressure estimation (SHAPE) is a novel method of dynamic contrast-enhanced (DCE) ultrasonography using encapsulated microbubble contrast material, which preferentially changes its acoustic behavior in response to ambient fluid pressure in the hepatic vessels and allows a noninvasive estimate of HVPG [77]. In a pilot study of 45 patients with variable liver diseases including 13 cases of NASH, clinically significant PHT was identified by SHAPE with a sensitivity of $89 \%$ and a specificity of $88 \%$, while correlation with fibrosis scores was less robust [77]. Another remarkable diagnostic approach applies the graph theory to DCE ultrasonography. Construction of a hepatic "vascular connectome" from the imaging information of microbubble disruption captured in tiny areas of $3 \times 3$ pixels allows a network analysis of reperfusion heterogeneity in the cirrhotic liver [78]. The degree by which vascular connectivity correlates with subtle changes in sinusoidal hemodynamics of the noncirrhotic liver and early increase in PVP remains to be seen. A great advantage of DCE ultrasonography methods is that they reflect on both structural and dynamic components of sinusoidal microcirculation.

Recent studies indicate that several magnetic resonance (MR)-based methods show excellent correlation with HVPG measurements even in the range of mild PHT. Thus, quantitative MR measures of liver $\mathrm{T}_{1}$ longitudinal relaxation time and splenic artery velocity may accurately estimate HVPG values on a continuous scale in patients with cirrhosis and PVP ranging from normal to the level of clinically significant PHT [79]. Moreover, liver stiffness measured by 2-dimensional (gradient-recalled echo) MR elastography showed good correlation with portal venous pressure in a small cohort of patients with cirrhosis with various etiologies including steatohepatitis and HVPG ranging from as low as 3-16 $\mathrm{mm} \mathrm{Hg}$ [80].

Another promising approach to extend PHT assessment to noncirrhotic individuals may be translated from animal studies that suggest the feasibility of direct PVP measurements guided by endoscopic ultrasound $[81,82]$. This technique has been validated by parallel HVPG readings in both normal and elevated ranges of PVP [81]. Moreover, use of a 19-gauge needle preloaded with a digital pressure wire provides additional ease and precision [82]. The analog device is slated for FDA approval in early 2018. It remains to be seen if any of these or other emerging methods of "endo-hepatology" [83] will allow the detection of mildly increased PVP in lieu of HVPG measurements, which could help define early vascular biomarkers of disease progression and identify patients at increased risk for adverse outcomes in NAFLD.

\section{Therapeutic Perspectives}

Current pharmaceutical options for the management of PHT and its acute complications such as variceal bleeding include the use of nonselective beta blockers and other 
Table 1 Established and emerging techniques for the assessment of portal hypertension

\begin{tabular}{|c|c|c|}
\hline Approach & Method & Comment \\
\hline \multirow[t]{7}{*}{ Intravascular } & Hepatic venous pressure gradient (HVPG) & Current gold standard for PVP measurement \\
\hline & & Allows simultaneous liver biopsy \\
\hline & & Invasive and performed in specialized centers only \\
\hline & & Less reliable in pre-sinusoidal PHT [74] \\
\hline & EUS-guided portal pressure monitoring & Provides direct access to the portal vein $[81,82]$ \\
\hline & & Allows simultaneous liver biopsy [107] \\
\hline & & FDA approval for analog device expected in $2 / 2018$ \\
\hline \multirow[t]{4}{*}{ Serum-based } & $\begin{array}{l}\text { Platelet count, AST-to-platelet ratio index, FIB-4 index, } \\
\text { Lok index, King's score }\end{array}$ & Primarily aimed at noninvasive fibrosis evaluation \\
\hline & & Potential as first-line screening for PHT [108] \\
\hline & & Established predictors of varices in upper GI bleeding [109] \\
\hline & Osteopontin, von Willebrand factor & Emerging predictors of survival in clinically significant PHT $[110,111]$ \\
\hline \multirow[t]{7}{*}{ Elastography } & Ultrasound-based elastography & Best utilized in ruling out advanced fibrosis [112-114] \\
\hline & Vibration-controlled transient elastography (VCTE) & Reasonable correlation with HVPG $[76,115,116]$ \\
\hline & Shear wave elastography (SWE) & VCTE values are low in noncirrhotic portal fibrosis [117] \\
\hline & Acoustic radiation force impulse imaging (ARFI) & Spleen stiffness may differentiate large versus small varices [118] \\
\hline & Magnetic resonance (MR)-based elastography (MRE) & Highly accurate noninvasive test for fibrosis $[119,120]$ \\
\hline & & $\begin{array}{l}\text { 2D (gradient-recalled echo) MRE and 3D (spin-echo echo-planar imag- } \\
\text { ing) MRE have comparable performance [121] }\end{array}$ \\
\hline & & $\begin{array}{l}\text { Good correlation with HVPG across a wide range [80] High costs limit } \\
\text { wide utilization }\end{array}$ \\
\hline \multirow[t]{2}{*}{ Endoscopy } & EGD & $\begin{array}{l}\text { Routine method for screening and management of varices in clinically } \\
\text { significant PHT }\end{array}$ \\
\hline & Probe-based confocal laser endomicroscopy & $\begin{array}{l}\text { Reveals early microcirculatory and structural changes in duodenal } \\
\text { mucosa associated with PHT [68] }\end{array}$ \\
\hline \multirow[t]{6}{*}{ Imaging } & Doppler ultrasonography & Easy, noninvasive tests for altered liver hemodynamics $[27,75]$ \\
\hline & Dynamic contrast-enhanced ultrasonography (DCE) & $\begin{array}{l}\text { Subharmonic gradient shows good correlation with HVPG in clinically } \\
\text { significant PHT [77] }\end{array}$ \\
\hline & Subharmonic aided pressure estimation (SHAPE) & $\begin{array}{l}\text { Automated graph analysis of the "vascular connectome" reveals loss of } \\
\text { synchronous reperfusion in cirrhosis, reflecting both structural and } \\
\text { dynamic derangements linked to PHT [78] }\end{array}$ \\
\hline & $\begin{array}{l}\text { Network connectivity analysis of DCE clusters of } \\
\text { liver }\end{array}$ & \\
\hline & Contrast-enhanced computer tomography & Detects varices and other portosystemic collaterals [122] \\
\hline & Multi-parametric MR & Combined assessment of inflammation and fibrosis [123] \\
\hline
\end{tabular}

vasoconstrictor drugs such as vasopressin and octreotide [8, 84]. In recent years, several new pharmacological strategies have been considered for the improved control of clinically significant PHT, with statins being best evaluated for their impact on PVP and survival in cirrhosis [85, 86]. However, most drug candidates implicated in the control of PHT have not yet reached the human phase of investigation and we only have experimental evidence for their PVP lowering effects (Table 2). Details about the mechanisms of action and potential utility of these agents have been the subject of several recent reviews [87-90]. From the viewpoint of noncirrhotic NAFLD, interventions that target reversible components of endothelial dysfunction and deranged sinusoidal circulation may be of particular interest. Prevention of HSC activation as a main mechanism of action in the management of PHT has been reported for several pharmaceutical agents. Administration of simvastatin in cirrhotic patients with an HVPG $\geq 12 \mathrm{~mm} \mathrm{Hg}$ significantly decreased IHVR, attenuated postprandial increase in HVPG, and improved effective liver perfusion and metabolic capacity $[85,91]$. These effects of statins may stem from increased eNOS activity by LSECs and from inhibition of Rho-kinase-mediated contractility of HSCs [92], which may shape the early phases of PHT. Further studies may determine whether some of the clinical benefits of statins related to cardiovascular and liver-related mortality in NAFLD [93, 94] originate from a direct impact on sinusoidal homeostasis. Additional agents with impact on HSC activation and contractility include mitochondrial antioxidants [95, 96], renin-angiotensin system inhibitors [97], and relaxin [98]. Finally, selective 
Table 2 Current and proposed options for the pharmaceutical management of portal hypertension

\begin{tabular}{lll}
\hline Drugs & Status & Mechanism of action and major observations
\end{tabular}

Nonselective beta blockers

Marketed

Other vasoconstrictors

Statins

NO modulators

Endothelin receptor antagonists

Selective $\beta_{3}$ receptor agonists

Relaxin

Renin-angiotensin system regulators

Angiogenesis inhibitors

Anti-inflammatory drugs

FXR agonists

TGR5 agonists

PPAR- $\alpha$ agonists

Antioxidants

Metformin

Anticoagulants

Antibiotics
Marketed Vasopressin, terlipressin, and octreotide are mainly utilized for acute reduction in PVP through splanchnic vasoconstriction and reduced portal inflow [84]

Phase III Beneficial effects observed on HVPG in cirrhotic patients [85, 91] involve enhanced eNOS expression and Akt-dependent phosphorylation [126], limited activity of Rho-kinase and its association with Ras [92] and upregulation of KLF2 under shear stress conditions [127], which may decrease endothelial dysfunction, HSC contractility and angiogenesis

Phase II Blocking phosphodiesterase 5 (PDE5) by vardenafil to prevent the breakdown of NO mediator cGMP was associated with reduced HVPG in a pilot human study [128]

Phase II Use of $\mathrm{ET}_{\mathrm{A}}$ and/or $\mathrm{ET}_{\mathrm{B}}$ receptor inhibitors (e.g., ambrisentan, bosentan, macitentan) is established in the treatment of pulmonary hypertension, but efficacy in PHT by preventing HSC contractility and fibrosis remains controversial [90]

Experimental Stimulation of $\beta_{3}$ receptors by CGP12177A and SR58611A causes relaxation of HSCs via cAMP accumulation and Rho-kinase inhibition in experimental cirrhosis [99]. No effect on normal PVP, indicating dynamic component of IHVR as major target [100]

Experimental cAMP-mediated actions of insulin-like peptide hormone relaxin on the RXFP1 receptor improve intrahepatic NO signaling and inhibit endothelin and angiotensin-II pathways to reduce HSC contractility in experimental PHT [98]

Experimental Inhibition of downstream renin-angiotensin system mediators Rho-kinase and Januskinase-2 prevents activation of HSCs and reduces PVP in experimental PHT [97]

Experimental Adenovirus-mediated dominant-negative PDGF receptor gene transfer prevents HSC activation associated with decreased PVP and liver fibrosis in experimental cirrhosis [129]

Experimental Inhibition of cyclooxygenase (COX) by celecoxib or use of terutroban, a thromboxaneA2/prostaglandin endoperoxide receptor antagonist, reduces inflammation, fibrosis, and angiogenesis by eliminating the effect of vasoactive eicosanoids in experimental PHT $[130,131]$

Experimental Complex FXR-mediated effects include reduced steatosis through VLDL clearance and inhibition of de novo lipogenesis, inhibition of liver inflammatory response by limiting COX-2, NF- $\mathrm{KB}$ and SOCS3 signaling, improving gut barrier function and dysbiosis [132-134]. FXR increases the expression of dimethylarginine dimethylaminohydrolase (DDAH), which eliminates asymmetric dimethylarginine (ADMA), a competitive endogenous inhibitor of endothelial nitric oxide synthase (eNOS) abundant in cirrhosis [102]

Steroidal FXR agonist OCA reduces experimental PHT by DDAH and eNOS upregulation and by Rho-kinase repression [103]. OCA and nonsteroidal FXR agonist PX20606 improves experimental PHT by reducing sinusoidal dysfunction, liver fibrosis, and vascular remodeling [105]

Experimental TGR5 has anti-inflammatory effects by inhibiting NF- $\mathrm{BB}$ and cytokine release, and the dual FXR/TGR5 agonist (INT-767) decreases steatosis, limits cytokine release, and promotes M2 polarization of macrophages [106]

Experimental Fenofibrate lowers PVP in experimental cirrhosis in association with improved endothelial function through increased NO bioavailability, reduced leukocyte recruitment, and reduced COX-1 expression and thromboxane production [135]

Experimental Reduced mitochondrial oxidative damage and ROS-mediated signaling pathways have been associated with increased NO availability in LSECs, deactivation of HSCs, and reduced fibrosis resulting in lower PVP in experimental cirrhosis upon administration of mitoquinone [96], cerium oxide nanoparticles [95], and the MnSOD mimetic Tempol [136]

Experimental Use of metformin results in improved liver NO levels, inhibition of Rho-kinase and stronger antioxidant effects associated with diminished activation of HSCs, less angiogenesis and inflammation, and lower PVP in experimental cirrhosis [137]

Experimental Rivaroxaban, a direct factor Xa inhibitor, reduces the frequency of sinusoid microthrombosis and decreases PVP in experimental cirrhosis [138]

Experimental Intestinal decontamination by rifaximin blocks TLR4-mediated activation of HSCs and modulates FXR signaling by changing intestinal bile acid composition associated with reduced fibrosis, angiogenesis, and PVP in experimental PHT [69] 
beta3 receptor agonists induce relaxation of HSCs via cAMP accumulation and Rho-kinase inhibition with no effect on normal PVP, indicating that these drugs primarily target the dynamic components of IHVR $[99,100]$.

FXR has recently become a major pharmacological target in NAFLD owing to its complex role in bile acid and lipid metabolism, inflammation, and fibrosis [101]. FXR increases the expression of dimethylarginine dimethylaminohydrolase (DDAH), which eliminates asymmetric dimethylarginine (ADMA), a competitive endogenous inhibitor of endothelial nitric oxide synthase (eNOS) abundant in cirrhosis [102]. Obeticholic acid (OCA), a synthetic bile acid ligand of FXR, reduces experimental PHT by reactivating FXR signaling pathways of vasorelaxation through DDAH and eNOS upregulation and Rho-kinase repression [103]. FXR stimulation also inhibits contraction of HSCs mediated by endothelin-1 [104]. The novel nonsteroidal FXR agonist PX20606 improves sinusoidal flow and lowers PVP in association with limiting fibrosis, angiogenesis, and endothelial dysfunction in experimental models of PHT [105]. Moreover, treatment of $\mathrm{db} / \mathrm{db}$ obese mice with INT-767, a dual FXR/TGR5 agonist, results in diminished hepatic steatosis, reduced expression of pro-inflammatory cytokines, and phenotype shift of macrophages toward the anti-inflammatory M2 phenotype [106]. Future studies will define the clinical utility of these and other emerging drug candidates in managing PVP that may not reach the level of clinical significance but could promote the progression of fibrosis and contribute to adverse outcomes in NAFLD.

\section{Conclusions}

We have now evidence that PHT develops in noncirrhotic NAFLD. IHVR is often elevated in NAFLD when steatosis is the only histological feature, and the subsequent increase in PVP can eventually amount to clinically significant PHT before cirrhosis is established. Narrowing of the sinusoidal space by steatotic hepatocytes is probably the earliest cause of shear stress as a major impetus for endothelial dysfunction, aggravated by additional biomechanical and rheological factors (ballooning, contractility, cell adhesion, capillarization, neovascularization, and extracellular matrix deposition) that may precede fibrosis. While clinically significant PHT in noncirrhotic NAFLD is rare and the impact of mild PHT on liver-related morbidity remains unclear, it is reasonable to speculate that sinusoidal pressure is a major factor of the pathobiology and any degree of increased PVP may contribute to disease progression by accelerating liver cell injury, inflammation, angiogenesis, and fibrosis. Improved methods of noninvasive PVP monitoring may further explore the pathogenesis NAFLD and better identify patients at increased risk of disease progression who would benefit from novel preventive and therapeutic strategies.

\section{Compliance with ethical standards}

Conflict of interest Nothing to report.

\section{References}

1. Loomba R, Sanyal AJ. The global NAFLD epidemic. Nat Rev Gastroenterol Hepatol. 2013;10:686-690.

2. Younossi ZM, Koenig AB, Abdelatif D, Fazel Y, Henry L, Wymer M. Global epidemiology of nonalcoholic fatty liver disease-meta-analytic assessment of prevalence, incidence, and outcomes. Hepatology. 2016;64:73-84.

3. Kim CH, Younossi ZM. Nonalcoholic fatty liver disease: a manifestation of the metabolic syndrome. Cleve Clin J Med. 2008;75:721-728.

4. Goh GB, McCullough AJ. Natural history of nonalcoholic fatty liver disease. Dig Dis Sci. 2016;61:1226-1233. https://doi. org/10.1007/s10620-016-4095-4.

5. Dulai PS, Singh S, Patel J, et al. Increased risk of mortality by fibrosis stage in nonalcoholic fatty liver disease: systematic review and meta-analysis. Hepatology. 2017;65:1557-1565.

6. Hagstrom H, Nasr P, Ekstedt M, et al. Fibrosis stage but not NASH predicts mortality and time to development of severe liver disease in biopsy-proven NAFLD. J Hepatol. 2017;67:1265-1273.

7. Bosch J, Garcia-Pagan JC. Complications of cirrhosis. I. Portal hypertension. J Hepatol. 2000;32:141-156.

8. Sanyal AJ, Bosch J, Blei A, Arroyo V. Portal hypertension and its complications. Gastroenterology. 2008;134:1715-1728.

9. Francque S, Verrijken A, Mertens I, et al. Noncirrhotic human nonalcoholic fatty liver disease induces portal hypertension in relation to the histological degree of steatosis. Eur J Gastroenterol Hepatol. 2010;22:1449-1457.

10. Mendes FD, Suzuki A, Sanderson SO, Lindor KD, Angulo P. Prevalence and indicators of portal hypertension in patients with nonalcoholic fatty liver disease. Clin Gastroenterol Hepatol. 2012;10:e1022.

11. Puoti C, Bellis L. Steatosis and portal hypertension. Eur Rev Med Pharmacol Sci. 2005;9:285-290.

12. Mueller S. Does pressure cause liver cirrhosis? The sinusoidal pressure hypothesis. World J Gastroenterol. 2016;22:10482-10501.

13. Vollmar B, Menger MD. The hepatic microcirculation: mechanistic contributions and therapeutic targets in liver injury and repair. Physiol Rev. 2009;89:1269-1339.

14. Nakata K, Leong GF, Brauer RW. Direct measurement of blood pressures in minute vessels of the liver. Am J Physiol. 1960;199:1181-1188.

15. Oda M, Yokomori H, Han JY. Regulatory mechanisms of hepatic microcirculation. Clin Hemorheol Microcirc. 2003;29:167-182.

16. Sarin SK, Kapoor D. Non-cirrhotic portal fibrosis: current concepts and management. J Gastroenterol Hepatol. 2002; 17:526-534.

17. Strauss E, Valla D. Non-cirrhotic portal hypertension-concept, diagnosis and clinical management. Clin Res Hepatol Gastroenterol. 2014;38:564-569.

18. Paton A, Reynolds TB, Sherlock S. Assessment of portal venous hypertension by catheterisation of hepatic vein. Lancet. 1953;1:918-921. 
19. Bosch J, Garcia-Pagan JC, Berzigotti A, Abraldes JG. Measurement of portal pressure and its role in the management of chronic liver disease. Semin Liver Dis. 2006;26:348-362.

20. Suk KT. Hepatic venous pressure gradient: clinical use in chronic liver disease. Clin Mol Hepatol. 2014;20:6-14.

21. Abraldes JG, Sarlieve P, Tandon P. Measurement of portal pressure. Clin Liver Dis. 2014;18:779-792.

22. Silva-Junior G, Baiges A, Turon F, et al. The prognostic value of hepatic venous pressure gradient in patients with cirrhosis is highly dependent on the accuracy of the technique. Hepatology. 2015;62:1584-1592.

23. Wada K, Fujimoto K, Fujikawa Y, Shibayama Y, Mitsui H, Nakata K. Sinusoidal stenosis as the cause of portal hypertension in choline deficient diet induced fatty cirrhosis of the rat liver. Acta Pathol Jpn. 1974;24:207-217.

24. Sun CK, Zhang XY, Wheatley AM. Increased NAD(P)H fluorescence with decreased blood flow in the steatotic liver of the obese Zucker rat. Microvasc Res. 2003;66:15-21.

25. Seifalian AM, Piasecki C, Agarwal A, Davidson BR. The effect of graded steatosis on flow in the hepatic parenchymal microcirculation. Transplantation. 1999;68:780-784.

26. Balci A, Karazincir S, Sumbas H, Oter Y, Egilmez E, Inandi T. Effects of diffuse fatty infiltration of the liver on portal vein flow hemodynamics. J Clin Ultrasound. 2008;36:134-140.

27. Hirooka M, Koizumi Y, Miyake T, et al. Nonalcoholic fatty liver disease: portal hypertension due to outflow block in patients without cirrhosis. Radiology. 2015;274:597-604.

28. Vonghia L, Magrone T, Verrijken A, et al. Peripheral and hepatic vein cytokine levels in correlation with non-alcoholic fatty liver disease (NAFLD)-related metabolic, histological, and haemodynamic features. PLoS One. 2015;10:e0143380.

29. Ijaz S, Yang W, Winslet MC, Seifalian AM. Impairment of hepatic microcirculation in fatty liver. Microcirculation. 2003; 10:447-456.

30. Chalasani N, Wilson L, Kleiner DE, et al. Relationship of steatosis grade and zonal location to histological features of steatohepatitis in adult patients with non-alcoholic fatty liver disease. J Hepatol. 2008;48:829-834.

31. Hijmans BS, Grefhorst A, Oosterveer MH, Groen AK. Zonation of glucose and fatty acid metabolism in the liver: mechanism and metabolic consequences. Biochimie. 2014;96:121-129.

32. Duwaerts CC, Maher JJ. Mechanisms of liver injury in nonalcoholic steatohepatitis. Curr Hepatol Rep. 2014;13:119-129.

33. Caldwell S, Lackner C. Perspectives on NASH histology: cellular ballooning. Ann Hepatol. 2017;16:182-184.

34. Straub BK, Stoeffel P, Heid H, Zimbelmann R, Schirmacher P. Differential pattern of lipid droplet-associated proteins and de novo perilipin expression in hepatocyte steatogenesis. Hepatology. 2008;47:1936-1946.

35. Caldwell S, Ikura Y, Dias D, et al. Hepatocellular ballooning in NASH. J Hepatol. 2010;53:719-723.

36. Rangwala F, Guy CD, Lu J, et al. Increased production of sonic hedgehog by ballooned hepatocytes. J Pathol. 2011;224:401-410.

37. Poisson J, Lemoinne S, Boulanger C, et al. Liver sinusoidal endothelial cells: physiology and role in liver diseases. $J$ Hepatol. 2017;66:212-227.

38. Shah V, Haddad FG, Garcia-Cardena G, et al. Liver sinusoidal endothelial cells are responsible for nitric oxide modulation of resistance in the hepatic sinusoids. J Clin Invest. 1997;100:2923-2930.

39. Aird WC. Phenotypic heterogeneity of the endothelium: II. Representative vascular beds. Circ Res. 2007;100:174-190.

40. McCuskey RS. Morphological mechanisms for regulating blood flow through hepatic sinusoids. Liver. 2000;20:3-7.
41. Pasarin M, La Mura V, Gracia-Sancho J, et al. Sinusoidal endothelial dysfunction precedes inflammation and fibrosis in a model of NAFLD. PLoS One. 2012;7:e32785.

42. Friedman SL. Hepatic stellate cells: protean, multifunctional, and enigmatic cells of the liver. Physiol Rev. 2008;88:125-172.

43. Marrone G, Shah VH, Gracia-Sancho J. Sinusoidal communication in liver fibrosis and regeneration. J Hepatol. 2016;65:608-617.

44. Bhathal PS, Grossman HJ. Reduction of the increased portal vascular resistance of the isolated perfused cirrhotic rat liver by vasodilators. J Hepatol. 1985;1:325-337.

45. Baffy G. Kupffer cells in non-alcoholic fatty liver disease: the emerging view. J Hepatol. 2009;51:212-223.

46. Szabo G, Dolganiuc A, Mandrekar P. Pattern recognition receptors: a contemporary view on liver diseases. Hepatology. 2006;44:287-298.

47. Moschen AR, Kaser S, Tilg H. Non-alcoholic steatohepatitis: a microbiota-driven disease. Trends Endocrinol Metab. 2013;24:537-545.

48. Arab JP, Martin-Mateos RM, Shah VH. Gut-liver axis, cirrhosis and portal hypertension: the chicken and the egg. Hepatol Int. 2017. https://doi.org/10.1007/s12072-017-9798-x.

49. Maher JJ, Leon P, Ryan JC. Beyond insulin resistance: innate immunity in nonalcoholic steatohepatitis. Hepatology. 2008;48:670-678.

50. Schaffner F, Popper H. Capillarization of hepatic sinusoids in man. Gastroenterology. 1963;44:239-242.

51. Kietzmann T, Gorlach A. Reactive oxygen species in the control of hypoxia-inducible factor-mediated gene expression. Semin Cell Dev Biol. 2005;16:474-486.

52. Miyao M, Kotani H, Ishida T, et al. Pivotal role of liver sinusoidal endothelial cells in NAFLD/NASH progression. Lab Invest. 2015;95:1130-1144.

53. Brunt EM, Janney CG, Di Bisceglie AM, Neuschwander-Tetri BA, Bacon BR. Nonalcoholic steatohepatitis: a proposal for grading and staging the histological lesions. Am J Gastroenterol. 1999;94:2467-2474.

54. Yeh MM, Brunt EM. Pathology of nonalcoholic fatty liver disease. Am J Clin Pathol. 2007;128:837-847.

55. Nagula S, Jain D, Groszmann RJ, Garcia-Tsao G. Histologicalhemodynamic correlation in cirrhosis-a histological classification of the severity of cirrhosis. J Hepatol. 2006;44:111-117.

56. Coulon S, Legry V, Heindryckx F, et al. Role of vascular endothelial growth factor in the pathophysiology of nonalcoholic steatohepatitis in two rodent models. Hepatology. 2013;57:1793-1805.

57. Iwakiri Y, Shah V, Rockey DC. Vascular pathobiology in chronic liver disease and cirrhosis-current status and future directions. $J$ Hepatol. 2014;61:912-924.

58. Saxena R, Theise ND, Crawford JM. Microanatomy of the human liver-exploring the hidden interfaces. Hepatology. 1999;30:1339-1346.

59. McCuskey RS. A dynamic and static study of hepatic arterioles and hepatic sphincters. Am J Anat. 1966;119:455-477.

60. Gill RM, Belt P, Wilson L, Bass NM, Ferrell LD. Centrizonal arteries and microvessels in nonalcoholic steatohepatitis. Am J Surg Pathol. 2011;35:1400-1404.

61. Povero D, Eguchi A, Niesman IR, et al. Lipid-induced toxicity stimulates hepatocytes to release angiogenic microparticles that require Vanin-1 for uptake by endothelial cells. Sci Signal. 2013;6:88.

62. Laleman W, Landeghem L, Wilmer A, Fevery J, Nevens F. Portal hypertension: from pathophysiology to clinical practice. Liver Int. 2005;25:1079-1090.

63. Francque $S$, Wamutu $S$, Chatterjee $S$, et al. Non-alcoholic steatohepatitis induces non-fibrosis-related portal hypertension associated with splanchnic vasodilation and signs of a 
hyperdynamic circulation in vitro and in vivo in a rat model. Liver Int. 2010;30:365-375.

64. Boursier J, Diehl AM. Nonalcoholic fatty liver disease and the gut microbiome. Clin Liver Dis. 2016;20:263-275.

65. Jiang C, Xie C, Li F, et al. Intestinal farnesoid X receptor signaling promotes nonalcoholic fatty liver disease. J Clin Invest. 2015; 125:386-402.

66. Parseus A, Sommer N, Sommer F, et al. Microbiota-induced obesity requires farnesoid X receptor. Gut. 2017;66:429-437.

67. Luther J, Garber JJ, Khalili H, et al. Hepatic injury in nonalcoholic steatohepatitis contributes to altered intestinal permeability. Cell Mol Gastroenterol Hepatol. 2015;1:222-232.

68. Rodriguez-Diaz E, Baffy G, Singh SK. Probe-based confocal laser endomicroscopy quantitative morphometric markers associated with portal hypertension in duodenal mucosa. Liver Int. 2016;36:223-231.

69. Zhu Q, Zou L, Jagavelu K, et al. Intestinal decontamination inhibits TLR4 dependent fibronectin-mediated cross-talk between stellate cells and endothelial cells in liver fibrosis in mice. J Hepatol. 2012;56:893-899.

70. Madsen BS, Havelund T, Krag A. Targeting the gut-liver axis in cirrhosis: antibiotics and non-selective beta-blockers. Adv Ther. 2013;30:659-670.

71. Wiest R, Albillos A, Trauner M, Bajaj JS, Jalan R. Targeting the gut-liver axis in liver disease. J Hepatol. 2017;67:1084-1103.

72. de Oliveira AC. Noninvasive assessment of portal hypertension and detection of esophageal varices in cirrhosis: state-of-the-art. Eur J Gastroenterol Hepatol. 2017;29:531.

73. Kim MY, Jeong WK, Baik SK. Invasive and non-invasive diagnosis of cirrhosis and portal hypertension. World J Gastroenterol. 2014;20:4300-4315.

74. Leung JC, Loong TC, Pang J, Wei JL, Wong VW. Invasive and non-invasive assessment of portal hypertension. Hepatol Int. 2017. https://doi.org/10.1007/s12072-017-9795-0.

75. Maruyama H, Yokosuka O. Ultrasonography for noninvasive assessment of portal hypertension. Gut Liver. 2017;11:464-473.

76. Roccarina D, Rosselli M, Genesca J, Tsochatzis EA. Elastography methods for the non-invasive assessment of portal hypertension. Expert Rev Gastroenterol Hepatol. 2017. https://doi. org/10.1080/17474124.2017.1374852.

77. Eisenbrey JR, Dave JK, Halldorsdottir VG, et al. Chronic liver disease: noninvasive subharmonic aided pressure estimation of hepatic venous pressure gradient. Radiology. 2013;268:581-588.

78. Amat-Roldan I, Berzigotti A, Gilabert R, Bosch J. Assessment of hepatic vascular network connectivity with automated graph analysis of dynamic contrast-enhanced US to evaluate portal hypertension in patients with cirrhosis: a pilot study. Radiology. 2015;277:268-276

79. Palaniyappan N, Cox E, Bradley C, et al. Non-invasive assessment of portal hypertension using quantitative magnetic resonance imaging. J Hepatol. 2016;65:1131-1139.

80. Gharib AM, Han MAT, Meissner EG, et al. Magnetic resonance elastography shear wave velocity correlates with liver fibrosis and hepatic venous pressure gradient in adults with advanced liver disease. Biomed Res Int. 2017;2017:2067479.

81. Huang JY, Samarasena JB, Tsujino T, Chang KJ. EUS-guided portal pressure gradient measurement with a novel 25-gauge needle device versus standard transjugular approach: a comparison animal study. Gastrointest Endosc. 2016;84:358-362.

82. Schulman AR, Thompson CC, Ryou M. EUS-guided portal pressure measurement using a digital pressure wire with realtime remote display: a novel, minimally invasive technique for direct measurement in an animal model. Gastrointest Endosc. 2016;83:817-820.
83. Chang KJ, Samarasena JB, Iwashita T, Nakai Y, Lee JG. Endohepatology: a new paradigm. Gastrointest Endosc Clin N Am. 2012;22:379-385.

84. de Franchis R, Baveno VIF. Expanding consensus in portal hypertension: report of the Baveno VI Consensus Workshop: Stratifying risk and individualizing care for portal hypertension. J Hepatol. 2015;63:743-752.

85. Abraldes JG, Albillos A, Banares R, et al. Simvastatin lowers portal pressure in patients with cirrhosis and portal hypertension: a randomized controlled trial. Gastroenterology. 2009;136:1651-1658.

86. Abraldes JG, Villanueva C, Aracil C, et al. Addition of simvastatin to standard therapy for the prevention of variceal rebleeding does not reduce rebleeding but increases survival in patients with cirrhosis. Gastroenterology. 2016;150:e1163.

87. Koyama Y, Xu J, Liu X, Brenner DA. New developments on the treatment of liver fibrosis. Dig Dis. 2016;34:589-596.

88. Nair H, Berzigotti A, Bosch J. Emerging therapies for portal hypertension in cirrhosis. Expert Opin Emerg Drugs. 2016;21:167-181.

89. Vargas JI, Arrese M, Shah VH, Arab JP. Use of statins in patients with chronic liver disease and cirrhosis: current views and prospects. Curr Gastroenterol Rep. 2017;19:43.

90. Schwabl P, Laleman W. Novel treatment options for portal hypertension. Gastroenterol Rep (Oxf). 2017;5:90-103.

91. Zafra C, Abraldes JG, Turnes J, et al. Simvastatin enhances hepatic nitric oxide production and decreases the hepatic vascular tone in patients with cirrhosis. Gastroenterology. 2004; 126:749-755.

92. Trebicka J, Hennenberg M, Laleman W, et al. Atorvastatin lowers portal pressure in cirrhotic rats by inhibition of RhoA/ Rho-kinase and activation of endothelial nitric oxide synthase. Hepatology. 2007;46:242-253.

93. Athyros VG, Alexandrides TK, Bilianou H, et al. The use of statins alone, or in combination with pioglitazone and other drugs, for the treatment of non-alcoholic fatty liver disease/ nonalcoholic steatohepatitis and related cardiovascular risk. An expert panel statement. Metabolism. 2017;71:17-32.

94. Kim RG, Loomba R, Prokop LJ, Singh S. Statin use and risk of cirrhosis and related complications in patients with chronic liver diseases: a systematic review and meta-analysis. Clin Gastroenterol Hepatol. 2017;15:e1528.

95. Oro D, Yudina T, Fernandez-Varo G, et al. Cerium oxide nanoparticles reduce steatosis, portal hypertension and display antiinflammatory properties in rats with liver fibrosis. $J$ Hepatol. 2016;64:691-698.

96. Vilaseca M, Garcia-Caldero H, Lafoz E, et al. Mitochondriatargeted antioxidant mitoquinone deactivates human and rat hepatic stellate cells and reduces portal hypertension in cirrhotic rats. Liver Int. 2017;37:1002-1012.

97. Klein S, Rick J, Lehmann J, et al. Janus-kinase-2 relates directly to portal hypertension and to complications in rodent and human cirrhosis. Gut. 2017;66:145-155.

98. Fallowfield JA, Hayden AL, Snowdon VK, et al. Relaxin modulates human and rat hepatic myofibroblast function and ameliorates portal hypertension in vivo. Hepatology. 2014;59:1492-1504.

99. Trebicka J, Hennenberg M, Schulze Probsting A, et al. Role of beta3-adrenoceptors for intrahepatic resistance and portal hypertension in liver cirrhosis. Hepatology. 2009;50:1924-1935.

100. Vasina V, Giannone F, Domenicali M, et al. Portal hypertension and liver cirrhosis in rats: effect of the beta3-adrenoceptor agonist SR58611A. Br J Pharmacol. 2012;167:1137-1147. 
101. Halilbasic E, Fuchs C, Traussnigg S, Trauner M. Farnesoid X receptor agonists and other bile acid signaling strategies for treatment of liver disease. Dig Dis. 2016;34:580-588.

102. Mookerjee RP, Mehta G, Balasubramaniyan V, et al. Hepatic dimethylarginine-dimethylaminohydrolase 1 is reduced in cirrhosis and is a target for therapy in portal hypertension. $J$ Hepatol. 2015;62:325-331.

103. Verbeke L, Farre R, Trebicka J, et al. Obeticholic acid, a farnesoid $\mathrm{X}$ receptor agonist, improves portal hypertension by two distinct pathways in cirrhotic rats. Hepatology. 2014;59:2286-2298.

104. Li J, Kuruba R, Wilson A, Gao X, Zhang Y, Li S. Inhibition of endothelin-1-mediated contraction of hepatic stellate cells by FXR ligand. PLoS One. 2010;5:e13955.

105. Schwabl P, Hambruch E, Seeland BA, et al. The FXR agonist PX20606 ameliorates portal hypertension by targeting vascular remodelling and sinusoidal dysfunction. J Hepatol. 2017;66:724-733.

106. McMahan RH, Wang XX, Cheng LL, et al. Bile acid receptor activation modulates hepatic monocyte activity and improves nonalcoholic fatty liver disease. J Biol Chem. 2013;288:11761-11770.

107. Schulman AR, Thompson CC, Odze R, Chan WW, Ryou M. Optimizing EUS-guided liver biopsy sampling: comprehensive assessment of needle types and tissue acquisition techniques. Gastrointest Endosc. 2017;85:419-426.

108. Wang L, Feng Y, Ma X, et al. Diagnostic efficacy of noninvasive liver fibrosis indexes in predicting portal hypertension in patients with cirrhosis. PLoS One. 2017;12:e0182969.

109. Rockey DC, Elliott A, Lyles T. Prediction of esophageal varices and variceal hemorrhage in patients with acute upper gastrointestinal bleeding. J Investig Med. 2016;64:745-751.

110. Bruha R, Jachymova M, Petrtyl J, et al. Osteopontin: a non-invasive parameter of portal hypertension and prognostic marker of cirrhosis. World J Gastroenterol. 2016;22:3441-3450.

111. Ferlitsch M, Reiberger T, Hoke M, et al. von Willebrand factor as new noninvasive predictor of portal hypertension, decompensation and mortality in patients with liver cirrhosis. Hepatology. 2012;56:1439-1447.

112. Wong VW, Vergniol J, Wong GL, et al. Diagnosis of fibrosis and cirrhosis using liver stiffness measurement in nonalcoholic fatty liver disease. Hepatology. 2010;51:454-462.

113. Cassinotto C, Boursier J, de Ledinghen V, et al. Liver stiffness in nonalcoholic fatty liver disease: a comparison of supersonic shear imaging, FibroScan, and ARFI with liver biopsy. Hepatology. 2016;63:1817-1827.

114. Vuppalanchi R, Siddiqui MS, Van Natta ML, et al. Performance characteristics of vibration-controlled transient elastography for evaluation of non-alcoholic fatty liver disease. Hepatology. 2018;67:134-144. https://doi.org/10.1002/hep.29489.

115. Bureau C, Metivier S, Peron JM, et al. Transient elastography accurately predicts presence of significant portal hypertension in patients with chronic liver disease. Aliment Pharmacol Ther. 2008;27:1261-1268.

116. Kumar A, Khan NM, Anikhindi SA, et al. Correlation of transient elastography with hepatic venous pressure gradient in patients with cirrhotic portal hypertension: a study of 326 patients from India. World J Gastroenterol. 2017;23:687-696.

117. Sharma P, Agarwal R, Dhawan S, et al. Transient elastography (Fibroscan) in patients with non-cirrhotic portal fibrosis. J Clin Exp Hepatol. 2017;7:230-234.

118. Sharma $P$, Kirnake V, Tyagi $P$, et al. Spleen stiffness in patients with cirrhosis in predicting esophageal varices. Am J Gastroenterol. 2013;108:1101-1107.

119. Loomba R, Wolfson T, Ang B, et al. Magnetic resonance elastography predicts advanced fibrosis in patients with nonalcoholic fatty liver disease: a prospective study. Hepatology. 2014;60:1920-1928.

120. Loomba R, Cui J, Wolfson T, et al. Novel 3D magnetic resonance elastography for the noninvasive diagnosis of advanced fibrosis in NAFLD: a prospective study. Am J Gastroenterol. 2016;111:986-994.

121. Shi Y, Xia F, Li QJ, et al. Magnetic resonance elastography for the evaluation of liver fibrosis in chronic hepatitis B and $\mathrm{C}$ by using both gradient-recalled echo and spin-echo echo planar imaging: a prospective study. Am J Gastroenterol. 2016;111:823-833.

122. Leung JC, Loong TC, Wei JL, et al. Histological severity and clinical outcomes of nonalcoholic fatty liver disease in nonobese patients. Hepatology. 2017;65:54-64.

123. Pavlides M, Banerjee R, Tunnicliffe EM, et al. Multiparametric magnetic resonance imaging for the assessment of non-alcoholic fatty liver disease severity. Liver Int. 2017;37:1065-1073.

124. Banares R, Moitinho E, Piqueras B, et al. Carvedilol, a new nonselective beta-blocker with intrinsic anti-alpha1-adrenergic activity, has a greater portal hypotensive effect than propranolol in patients with cirrhosis. Hepatology. 1999;30:79-83.

125. Villanueva C, Albillos A, Genesca J, et al. Development of hyperdynamic circulation and response to beta-blockers in compensated cirrhosis with portal hypertension. Hepatology. 2016;63:197-206.

126. Abraldes JG, Rodriguez-Vilarrupla A, Graupera M, et al. Simvastatin treatment improves liver sinusoidal endothelial dysfunction in CCl4 cirrhotic rats. J Hepatol. 2007;46:1040-1046.

127. Marrone G, Russo L, Rosado E, et al. The transcription factor KLF2 mediates hepatic endothelial protection and paracrine endothelial-stellate cell deactivation induced by statins. $J$ Hepatol. 2013;58:98-103.

128. Deibert P, Schumacher YO, Ruecker G, et al. Effect of vardenafil, an inhibitor of phosphodiesterase-5, on portal haemodynamics in normal and cirrhotic liver-results of a pilot study. Aliment Pharmacol Ther. 2006;23:121-128.

129. Reichenbach V, Fernandez-Varo G, Casals G, et al. Adenoviral dominant-negative soluble PDGFRbeta improves hepatic collagen, systemic hemodynamics, and portal pressure in fibrotic rats. J Hepatol. 2012;57:967-973.

130. Gao JH, Wen SL, Yang WJ, et al. Celecoxib ameliorates portal hypertension of the cirrhotic rats through the dual inhibitory effects on the intrahepatic fibrosis and angiogenesis. PLoS One. 2013;8:e69309.

131. Rosado E, Rodriguez-Vilarrupla A, Gracia-Sancho J, et al. Terutroban, a TP-receptor antagonist, reduces portal pressure in cirrhotic rats. Hepatology. 2013;58:1424-1435.

132. Wang YD, Chen WD, Wang M, Yu D, Forman BM, Huang W. Farnesoid $X$ receptor antagonizes nuclear factor kappaB in hepatic inflammatory response. Hepatology. 2008;48:1632-1643.

133. Xu Z, Huang G, Gong W, et al. FXR ligands protect against hepatocellular inflammation via SOCS3 induction. Cell Signal. 2012;24:1658-1664.

134. Verbeke L, Farre R, Verbinnen B, et al. The FXR agonist obeticholic acid prevents gut barrier dysfunction and bacterial translocation in cholestatic rats. Am J Pathol. 2015;185:409-419.

135. Rodriguez-Vilarrupla A, Lavina B, Garcia-Caldero $\mathrm{H}$, et al. PPARalpha activation improves endothelial dysfunction and reduces fibrosis and portal pressure in cirrhotic rats. J Hepatol. 2012;56:1033-1039.

136. Garcia-Caldero H, Rodriguez-Vilarrupla A, Gracia-Sancho J, et al. Tempol administration, a superoxide dismutase mimetic, reduces hepatic vascular resistance and portal pressure in cirrhotic rats. J Hepatol. 2011;54:660-665. 
137. Tripathi DM, Erice E, Lafoz E, et al. Metformin reduces hepatic resistance and portal pressure in cirrhotic rats. Am J Physiol Gastrointest Liver Physiol. 2015;309:G301-G309.

138. Vilaseca M, Garcia-Caldero H, Lafoz E, et al. The anticoagulant rivaroxaban lowers portal hypertension in cirrhotic rats mainly by deactivating hepatic stellate cells. Hepatology. 2017;65:2031-2044. 